\title{
THE COMPREHENSIVE FACTORIZATION OF A FUNCTOR
}

\author{
BY ROSS STREET AND R. F. C. WALTERS
}

Communicated by Saunders Mac Lane, March 12, 1973

In this article we show that every functor has a factorization into an initial functor followed by a discrete 0 -fibration and that this factorization is functorial. Size considerations will be ignored but may be easily filled in; we assume the existence of a category of sets large enough to dwarf any given finite number of categories.

There is an analogy between the category Set of sets and the category Cat of categories which is partly explained by the observation that each is a category of types for a suitable hyperdoctrine. A hyperdoctrine (Lawvere [4]) consists of a category $T$ of types and a functor $P: T^{\mathrm{op}} \rightarrow$ Cat satisfying conditions. The comprehension schema (also see [4]) is expressed by a pair of adjoint functors

$$
P X \leftrightarrows T / X
$$

for each object $X$ of $T$, where $T / X$ is the category of objects over $X$. It is often the case that this structure arises from more usual structure on the category $T$; namely:

(1) a factorization system $(E, M)$ on $T$;

(2) a category object $\Omega$ in $T$ which "classifies the $M$-subobjects".

The sense in which (1) is intended is that of Freyd-Kelly [2]. We say that $\Omega$ classifies the $M$-subobjects when there is a natural equivalence of categories $T(X, \Omega) \approx M(X)$, where $M(X)$ is the full subcategory of $T / X$ consisting of the arrows in $M$ with target $X$. Then $P X=T(X, \Omega)$. From (1), the functor "take the $(E, M)$-image" is the left adjoint of the inclusion $M(X) \rightarrow T / X$; this adjunction combines with (2) to yield the comprehension schema.

The familiar example of a hyperdoctrine which arises in the above way is provided by the power-set functor $P:$ Set $^{\text {op }} \rightarrow$ Cat. Here $M$ consists of monomorphisms, $E$ of epimorphisms, and $\Omega$ is a set with two elements. These considerations lie at the heart of the elementary theory of the category of sets in the new elegant form-elementary topos theory-due to Lawvere-Tierney (see Freyd [1]). A topos $T$ is a finitely complete, cartesian closed category satisfying (2) where $M$ consists of the mono-

AMS (MOS) subject classifications (1970). Primary 18A20; Secondary 18A25, 18A40, 18D05, 18D30.

Key words and phrases. Factorization of functors, initial functors, discrete fibrations, comprehension schema, comma categories, Kan extensions. 
morphisms. It can be proved that $(E, M)$ is a factorization system on $T$ where $E$ consists of the epimorphisms.

It is our aim to show here that there is a factorization system on Cat which gives rise to the hyperdoctrine with $T=$ Cat and $P X$ equal to the category of set-valued functors on $X$. Moreover, we show that the classes $E, M$ are already distinguished in the literature; namely, $E$ consists of the initial functors, $M$ consists of the discrete 0 -fibrations. Forthcoming papers (see [6]) on the elementary theory of the 2-category of categories will consider the abstraction of the concept of discrete 0 -fibration to an arbitrary 2-category and the corresponding condition (2).

For any category $A$, let $!: A \rightarrow \mathbf{1}$ denote the unique functor into the terminal object of Cat. Let $*: \mathbf{1} \rightarrow$ Set denote the constant functor at the one point set. For functors $A \rightarrow^{r} D, B \rightarrow^{s} D$ with the same codomain, the comma category $r / s$ is defined by the pullback

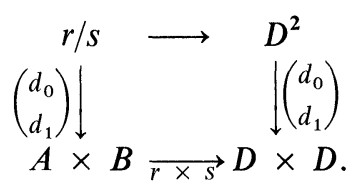

The natural transformation

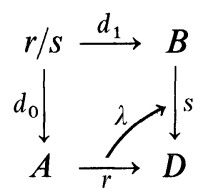

corresponding to $r / s \rightarrow D^{2}$ is characterized by the universal property that composition with $\lambda$ sets up a bijection

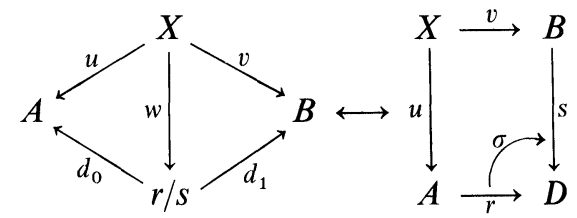

between arrows of spans $w$ and natural transformations $\sigma$. The particular case which is important here is the comma category $* / k$ for a functor $B \rightarrow{ }^{k}$ Set; the objects are pairs $(b, \xi)$ where $b$ is an object of $B$ and $\xi$ is an element of $k b$, and the arrows $(b, \xi) \rightarrow^{\beta}\left(b^{\prime}, \xi^{\prime}\right)$ are arrows $b \rightarrow^{\beta} b^{\prime}$ in $B$ satisfying $(k \beta) \xi=\xi^{\prime}$.

A natural transformation

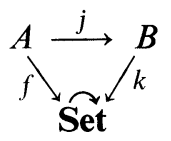

is said to exhibit $k$ as a left extension of $f$ along $j$ when it sets up a bijection 
between natural transformations

and natural transformations
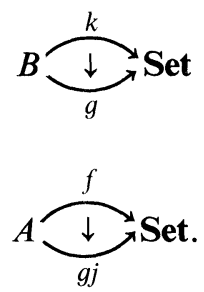

Recall also (see Mac Lane [5, p. 236]) the formula

$$
k b=\operatorname{colim}\left(j / b \stackrel{d_{0}}{\longrightarrow} A \stackrel{f}{\longrightarrow} \text { Set }\right) .
$$

Proposition 1. For any functor $B \rightarrow^{k}$ Set, the natural transformation

$$
!=d_{0} \downarrow_{1}^{* / k \stackrel{d_{1}}{\longrightarrow}} \stackrel{B}{\longrightarrow} \underset{\downarrow}{\longrightarrow} \text { Set }
$$

exhibits $k$ as a left extension of $*$ ! along $d_{1}$.

A functor $E \rightarrow^{p} B$ is called a discrete 0 -fibration when there exists a functor $B \rightarrow^{k}$ Set and an isomorphism $E \cong * / k$ such that

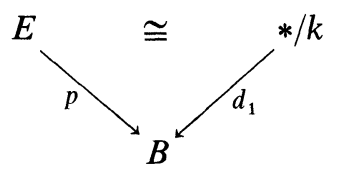

commutes. By Proposition $1, k$ is uniquely determined up to isomorphism by $p$.

A functor $A \rightarrow^{e} E$ is called initial when the canonical natural transformation

is an isomorphism.

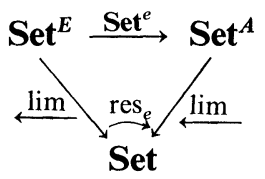

These two definitions can be made independent of the choice of the category Set. A functor $E \rightarrow^{p} B$ is called a 0 -fibration (Gray [3]) when the canonical functor $E^{2} \rightarrow p / B$, induced by the natural transformation

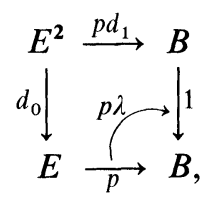


has a left adjoint with identity unit. The 0 -fibration $p$ is discrete if and only if, for each object $b$ in $B$, the category $E_{b}$, defined by the pullback

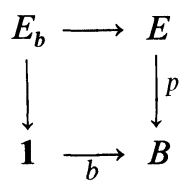

is discrete. This is easily proved by defining $B \rightarrow^{k}$ Set on objects by $k b=E_{b}$. That (a), (c) in the following proposition are equivalent is well known (see Mac Lane [5 p. 213] for the dual); we give a different proof. Condition (b) is the important one for us.

Proposition 2. The following conditions are equivalent:

(a) the functor $A \rightarrow^{e} E$ is initial;

(b) the identity natural transformation,

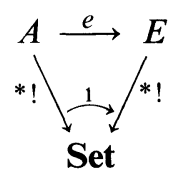

exhibits $*$ ! as a left extension of $*$ ! along $e$;

(c) for each object $x$ in $E$, the comma category $e / x$ is nonempty and pathwise connected.

Proof. Consider the natural transformation in the triangle

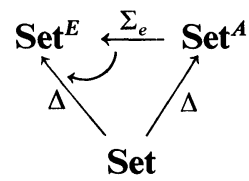

obtained from the triangle containing res $_{e}$ by replacing each functor by its left adjoint. Then $\Delta$ takes a set to the constant functor at that set, and $\Sigma_{e}$ takes a functor to its left extension along $e$. Also $e$ is initial if and only if $\Sigma_{e} \Delta \rightarrow \Delta$ is an isomorphism. Every constant functor $A \rightarrow$ Set is a coproduct of copies of the constant functor $*$ ! and $\Sigma_{e}$, being a left adjoint, preserves coproducts. So $\Sigma_{e} \Delta \rightarrow \Delta$ is an isomorphism if and only if $\Sigma_{e}(* !)=*$ ! . This proves (a) $\Leftrightarrow(\mathrm{b})$. From the construction of colimits in Set it is clear that (c) says precisely that, for each object $x$ in $E$,

$$
\operatorname{colim}(e / x \stackrel{!}{\longrightarrow} \mathbf{1} \stackrel{*}{\longrightarrow} \text { Set })
$$

is the one point set. Using the formula for left extensions, we see that this is equivalent to (b). 
THEOREM 3. Any functor $A \rightarrow^{f} B$ admits a factorization

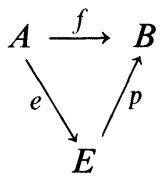

where $e$ is an initial functor and $p$ is a discrete 0-fibration.

Proof. Define $B \rightarrow^{k}$ Set to be the left extension of $A \rightarrow^{!} 1 \rightarrow^{*}$ Set along $f$. Then define $E \rightarrow^{p} B$ to be $* / k \rightarrow^{d_{1}} B$. So $p$ is a discrete 0 -fibration. The natural transformation

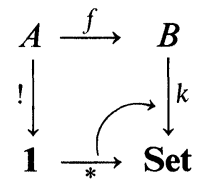

factors through the natural transformation

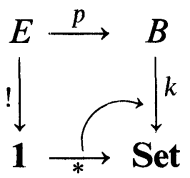

by the universal property of the latter. So we have a functor $e$ such that

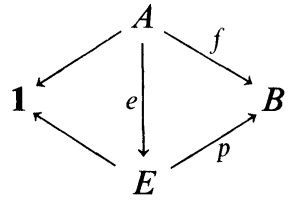

commutes. Consider the diagram

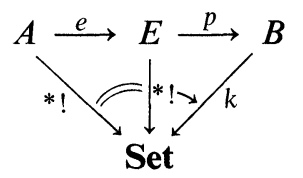

The outside triangle is a left extension by definition of $k$. The right triangle is a left extension by Proposition 1. So the left triangle is a left extension; $e$ is initial.

THEOREM 4. Suppose, in the commutative diagram

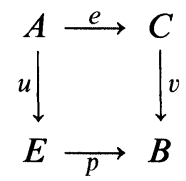


of functors, $e$ is initial and $p$ is a discrete 0-fibration. Then there exists a unique functor $C \rightarrow^{w} E$ such that $p w=v$ and $w e=u$.

Proof. Since $p$ is a discrete 0 -fibration there is a functor $k$ and a universal natural transformation

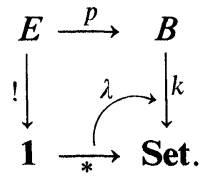

Since $e$ satisfies (b) of Proposition 2, composing with $e$ sets up a bijection

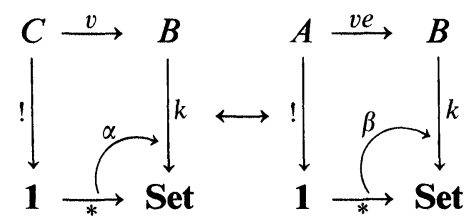

between natural transformations $\alpha$ and natural transformations $\beta$. By the universal property of $\lambda$, this implies that composing with $e$ sets up a bijection

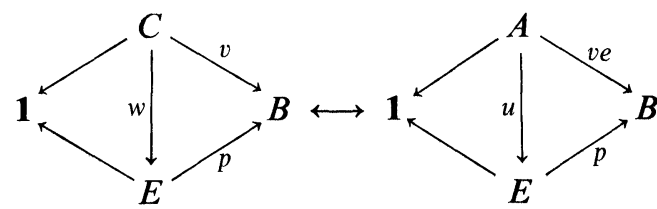

between arrows of spans $w$ and arrows of spans $u$.

It follows from Theorem 4 that the factorization of Theorem 3 is unique up to isomorphism and that the notion of image so obtained is functorial.

\section{REFERENCES}

1. P. J. Freyd, Aspects of topoi, Bull. Austral. Math. Soc. 7 (1972), 1-76.

2. P. J. Freyd and G. M. Kelly, Categories of continuous functors. I, J. Pure Appl. Algebra 2 (1972), 169-191.

3. J. W. Gray, The categorical comprehension scheme, Category Theory, Homology Theory and their Applications (Battelle Institute Conference, Seattle, Wash., 1968, vol. 3), Springer, Berlin, 1969, pp. 242-312. MR 40 \#2728.

4. F. W. Lawvere, Equality in hyperdoctrines and comprehension schema as an adjoint functor, Applications of Categorical Algebra, Proc. Sympos. Pure Math., vol. 17, Amer. Math. Soc., Providence, R.I., 1970, pp. 1-14. MR 41 \#1829.

5. S. Mac Lane, Categories for the working mathematician, Graduate Texts in Math., 5, Springer-Verlag, Berlin and New York, 1971.

6. R. H. Street, Elementary cosmoi, Proc. Sydney Category Seminar (1972), Lecture Notes in Math., Springer-Verlag, Berlin, 1973.

Department of Mathematics, Macquarie University, North Ryde 2113, Australia DePartment of Mathematics, University of Sydney, SydNEy 2006, Australia 